\title{
Universal Phase Shift and Nonexponential Decay of Driven Single-Spin Oscillations
}

\author{
F. H. L. Koppens, ${ }^{1}$ D. Klauser, ${ }^{2}$ W. A. Coish, ${ }^{2}$ K. C. Nowack, ${ }^{1}$ L. P. Kouwenhoven, ${ }^{1}$ D. Loss, ${ }^{2}$ and L. M. K. Vandersypen ${ }^{1}$ \\ ${ }^{1}$ Kavli Institute of NanoScience Delft, P.O. Box 5046, 2600 GA Delft, The Netherlands \\ ${ }^{2}$ Department of Physics, University of Basel, Klingelbergstrasse 82, CH-4056 Basel, Switzerland
}

(Received 24 March 2007; published 7 September 2007)

\begin{abstract}
We study, both theoretically and experimentally, driven Rabi oscillations of a single electron spin coupled to a nuclear-spin bath. Because of the long correlation time of the bath, two unusual features are observed in the oscillations. The decay follows a power law, and the oscillations are shifted in phase by a universal value of $\sim \pi / 4$. These properties are well understood from a theoretical expression that we derive here in the static limit for the nuclear bath. This improved understanding of the coupled electronnuclear system is important for future experiments using the electron spin as a qubit.
\end{abstract}

A quantum bit is engineered such that its coupling to the disturbing environment is minimized. Understanding and controlling this coupling is therefore a major subject in the field of quantum information processing. It is not solely the coupling strength but also the dynamics of the environment that governs the quantum coherence. In particular, the limit where these dynamics are slow compared to the evolution of the quantum system is interesting. The well-known Markovian Bloch equations that describe the dynamics of a driven system, including the exponential decay of the longitudinal and transverse magnetization [1], then lose their validity. Such deviations from the exponential behavior have been studied theoretically [2,3] and experimentally, for instance in superconducting qubit systems [4].

An electron spin confined in the solid state is affected predominantly by phonons via the spin-orbit interaction [5-9], and by nuclear spins in the host material via the hyperfine interaction. At low temperature, coupling to the nuclear spins is the dominant decoherence source [10-17]. Although this strong coupling leads to an apparent decoherence time $T_{2}^{*}$ of the order of $20 \mathrm{~ns}$ when time averaged over experimental runs, the decoherence time $T_{2}$ strongly depends on the dynamics in the nuclear-spin bath. This typical nuclear-spin dynamics is very slow, because the nuclear spins are only weakly coupled with each other and the bath itself is coupled very weakly to its dissipative environment (like phonons). This implies that here, the Markovian Bloch equations are not valid.

Here we study the dynamics and decoherence of an electron spin in a quantum dot that is coherently driven via pulsed magnetic resonance, and is coupled to a nuclearspin bath with a long correlation time. We find experimentally that, remarkably, the electron spin oscillates coherently, even when the Rabi period is much longer than $T_{2}^{*}=10-20 \mathrm{~ns}$. In addition, the characteristics of the driven electron-spin dynamics are unusual. The decay of the Rabi oscillations is not exponential but follows a power law and a universal (parameter independent) phase shift emerges. We compare these experimental results with a theoretical expression, derived in the limit of a static nuclear-spin bath.

We consider a double quantum dot with one electron in each dot and a static external magnetic field in the $z$ direction, resulting in a Zeeman splitting $\epsilon_{z}=g \mu_{B} B_{z}$. The spin transitions are driven by a burst of a transverse oscillating field along the $x$ direction with amplitude $B_{\text {ac }}$ and frequency $\omega$, which is generated by a current $I_{s}$ through a microfabricated wire close to the double dot [18]. The interaction between the electron spin and the nuclear bath is described by the Fermi contact hyperfine interaction $\vec{S} \cdot \vec{h}$, where $\vec{h}$ is the field generated by the nuclear spins at the position of the electron. For a large but finite number of nuclear spins $\left(N \sim 10^{6}\right.$ for lateral GaAs dots) $h_{z}$ is Gaussian distributed (due to the centrallimit theorem) with mean $h_{0}=\bar{h}_{z}$ and variance $\sigma^{2}=$ $\overline{\left(h_{z}-h_{0}\right)^{2}}$ [10-12]. For a sufficiently large external magnetic field $\left(\epsilon_{z} \gg \sigma\right)$, we may neglect the transverse terms $S_{\perp} \cdot h_{\perp}$ of the hyperfine interaction that give rise to electron-nuclear-spin flip-flops (see below). Furthermore, if the singlet-triplet energy splitting $J$ is much smaller than both $\epsilon_{z}$ and $g \mu_{B} B_{\text {ac }}$, we may treat the spin dynamics of the electrons in each dot independently (valid for times less than $1 / J)$.

For each dot we thus have the following spin Hamiltonian $(\hbar=1)$ :

$$
H(t)=\frac{1}{2}\left(\epsilon_{z}+h_{z}\right) \sigma_{z}+\frac{b}{2} \cos (\omega t) \sigma_{x}
$$

where $\sigma_{i}$ (with $i=x, z$ ) are the Pauli matrices and $b=$ $g \mu_{B} B_{\mathrm{ac}}$ (taken to be equal in both dots). Here, $h_{z}$ is considered as completely static during the electron-spin time evolution. This is justified because the correlation time of the fluctuations in the nuclear-spin system due to the dipole-dipole and hyperfine-mediated interaction between the nuclear spins, which is predicted to be $\gtrsim$ $10-100 \mu \mathrm{s}[10-12,19-23]$, is much larger than the time scale for electron-spin dynamics considered here (up to $1 \mu \mathrm{s})$. 
In the experiment, the electron-spin state is detected in a regime where electron transport through the double quantum dot occurs via transitions from spin states with one electron in each dot [denoted as $(1,1)]$ to the singlet state $|S(0,2)\rangle$ with two electrons in the right dot. These transitions, governed via the tunnel coupling $t_{c}$ by the tunneling Hamiltonian $H_{t_{c}}=t_{c}|S(1,1)\rangle\langle S(0,2)|+$ H.c., are only possible for antiparallel spins, because $\left\langle\uparrow \uparrow\left|H_{t_{c}}\right| S(0,2)\right\rangle=\left\langle\downarrow \downarrow\left|H_{t_{c}}\right| S(0,2)\right\rangle=0$, while $\left\langle\downarrow \uparrow\left|H_{t_{c}}\right| S(0,2)\right\rangle$, $\left\langle\uparrow \|\left|H_{t_{c}}\right| S(0,2)\right\rangle \neq 0$. Therefore, the states with even spin parity (parallel spins) block transport, while the states with odd spin parity (antiparallel spins) allow for transport. If the system is initialized to an even spin-parity state, the oscillating transverse magnetic field (if on resonance) rotates one (or both) of the two spins and thus lifts the blockade [18]. Initializing to $|\uparrow\rangle$ in both dots (the case with $|\downarrow\rangle$ gives the same result), we calculate the probability for an odd spin parity $P_{\text {odd }}$ under time evolution for each of the two spins governed by the Hamiltonian in Eq. (1).

Introducing the detuning from resonance $\delta_{\omega}=$ $\epsilon_{z}+h_{z}-\omega$, the probability of finding spin-up for a single value of $h_{z}$ in the rotating wave approximation (which is valid for $\left.\left(b / \epsilon_{z}\right)^{2} \ll 1\right)$ is given by

$$
\begin{aligned}
P_{\uparrow, \delta_{\omega}}(t)= & \frac{1}{2}\left[1+\frac{4 \delta_{\omega}^{2}}{b^{2}+4 \delta_{\omega}^{2}}+\frac{b^{2}}{b^{2}+4 \delta_{\omega}^{2}}\right. \\
& \left.\times \cos \left(\frac{t}{2} \sqrt{b^{2}+4 \delta_{\omega}^{2}}\right)\right] .
\end{aligned}
$$

Assuming that $\omega=h_{0}+\epsilon_{z}$, i.e., $\delta_{\omega}=h_{z}-h_{0}$, we find when averaging over the Gaussian distribution of $h_{z}$ values (see [24])

$$
P_{\uparrow}(t) \sim \frac{1}{2}+C+\sqrt{\frac{b}{8 \sigma^{2} t}} \cos \left(\frac{b}{2} t+\frac{\pi}{4}\right)+\mathcal{O}\left(\frac{1}{t^{3 / 2}}\right),
$$

for $\quad t \gg \max \left(\frac{1}{\sigma}, 1 / b, b / 2 \sigma^{2}\right)$, with $\quad C=\frac{1}{2}-\frac{\sqrt{2 \pi} b}{8 \sigma} \times$ $\exp \left(\frac{b^{2}}{8 \sigma^{2}}\right) \operatorname{erfc}\left(\frac{b}{2 \sqrt{2} \sigma}\right)$. We can now calculate the probability of finding an odd spin-parity state taking $\omega=h_{0}+\epsilon_{z}$ for both dots and drawing the value of $h_{z}$ independently from a distribution with width $\sigma$ in each dot:

$$
\begin{gathered}
P_{\text {odd }}(t)=P_{\uparrow, L}(t)\left[1-P_{\uparrow, R}(t)\right]+\left[1-P_{\uparrow, L}(t)\right] P_{\uparrow, R}(t) \\
=\frac{1}{2}-2 C^{2}-C \frac{f(t)}{\sqrt{t}}-\frac{g(t)}{t}+\mathcal{O}\left(\frac{1}{t^{3 / 2}}\right) ; \\
f(t)=\sqrt{\frac{2 b}{\sigma^{2}}} \cos \left(\frac{b t}{2}+\frac{\pi}{4}\right), \\
g(t)=\frac{b}{8 \sigma^{2}}\left[1+\cos \left(b t+\frac{\pi}{2}\right)\right] .
\end{gathered}
$$

This result is valid for times $t \gtrsim \max \left(1 / \sigma, 1 / b, b / 2 \sigma^{2}\right) \sim$ $20 \mathrm{~ns}$ for a $1.4 \mathrm{mT}$ nuclear field (see below) and $b \leq 2 \sigma$ (accessible experimental regime). The $1 / t$ term oscillates with the double Rabi frequency which is the result of both spins being rotated simultaneously (see also [18]). This term only becomes important for $b>\sigma$, because in that case for both spins most of the nuclear-spin distribution is within the Lorentzian line shape of the Rabi resonance. The $1 / \sqrt{t}$-term oscillates with the Rabi frequency and originates from only one of the two spins being rotated [18]. This term is important when $b<\sigma$, i.e., when only a small fraction of the nuclear-spin distribution is within the line shape of the Rabi resonance.

We also give the expression for $P_{\text {odd }}(t)$ for the case where only one of the two spins is on resonance $\left(\epsilon_{z}+h_{0}-\right.$ $\omega=0)$, while the other is far off resonance $\left(\mid \epsilon_{z}+h_{0}-\right.$ $\omega \mid \gg \sigma)$. In this case the spin in one dot always remains up while the spin in the other dot rotates. This leads to

$$
P_{\mathrm{odd}}^{(1)}(t)=1-P_{\uparrow}(t)=\frac{1}{2}-C-\frac{f(t)}{4 \sqrt{t}}+\mathcal{O}\left(\frac{1}{t^{3 / 2}}\right),
$$

with the same range of validity as in Eq. (4). We see that the $1 / t$ term, which oscillates with frequency $b$, is not present in this case.

The expressions for $P_{\text {odd }}(t)$ [Eqs. (4) and (7)] reveal two interesting features: the power-law decay and a universal phase shift of $\pi / 4$ [see Eq. (5)] in the oscillations which is independent of all parameters. These features can both only appear if the nuclear field $h_{z}$ is static during a time much longer than the Rabi period. This is crucial because only then the driven spin coherence for one fixed value of $h_{z}$ is fully preserved. Because different values of $h_{z}$ give different oscillation frequencies, the decay is due to averaging over the distribution in $h_{z}$.

The phase shift is closely related to the power-law decay because it also finds its origin in the off-resonant contributions. These contributions have a higher Rabi frequency and shift the average oscillation in phase. This universal phase shift therefore also characterizes the spin decay, together with the power law. Interestingly, the specific shape of the distribution in $h_{z}$ (as long as it is peaked around the resonance) is not crucial for the appearance of both the power-law decay and the phase shift [24]. The values of the decay power and the phase shift are determined by the dependence of the oscillation frequency on $h_{z}$ (in this case $\sqrt{b^{2}+4 \delta_{\omega}^{2}}$ ).

A power-law decay has previously been found theoretically in $[10,12,25,26]$ and both a power-law decay $\left(1 / t^{3 / 2}\right)$ and a universal phase shift also appear in double dot correlation functions [13,21]. In [17] a singlet-triplet correlation function was measured, but the amplitude of the oscillations was too small for the phase shift and the power-law decay to be determined. Here, we consider driven Rabi oscillations of a single electron spin with a power-law decay of $1 / \sqrt{t}$ that is already valid after a short time $1 / \sigma \sim 20 \mathrm{~ns}$. Therefore, the amplitude of the driven spin oscillations is still high when the power-law behavior sets in, even for small driving fields $(b<2 \sigma)$, which are experimentally easier to achieve. The power-law decay and the phase shift thus should be observable in the experiment. 
We now discuss the observation of the power-law decay in the experimental data of which a selection is shown in Fig. 1. The data are obtained with the same device and under the same experimental conditions as in [18]. A fit is carried out to the observed oscillations for four different driving fields $B_{\text {ac }}$ (Fig. 1), with three different fit functions: the theoretical expressions [Eqs. (4) and (7) with $b$ and a constant scaling factor as fit parameters] and an exponentially decaying cosine. The width of the nuclear distribution $\sigma=g \mu_{B}(1.4 \mathrm{mT})$ is obtained from a fit of the steadystate value $\frac{1}{2}-2 C^{2}$ of $P_{\text {odd }}(t)$ to a data set obtained at $t=$ 950 ns [Fig. 2(a)].

For the range $B_{\mathrm{ac}} \geq 1.9 \mathrm{mT}$, we find good agreement with the model that predicts a power-lay decay of $1 / \sqrt{t}$ [Eq. (4); $h_{0}$ equal for both dots], while the fit with an exponentially decaying cosine is poor (dotted lines in Fig. 1). The power of the decay is independently verified by means of a fit to the data with $a_{1}+a_{2} \cos \left(2 \pi t / a_{3}+\right.$ $\pi / 4) / t^{d}$ where, besides $a_{1,2,3}$, the power $d$ of the time $t$ is a fit parameter as well. We find values of $d \sim 0.6$ [Fig. 2(b)], close to the predicted $1 / \sqrt{t}$ dependence.

We see much better correspondence of the data with Eq. (4) than with Eq. (7), from which we can conclude that the mean of the Gaussian distribution $h_{0}$ is comparable for both dots (in equilibrium, we expect $h_{0} \sim 0$ in both dots).
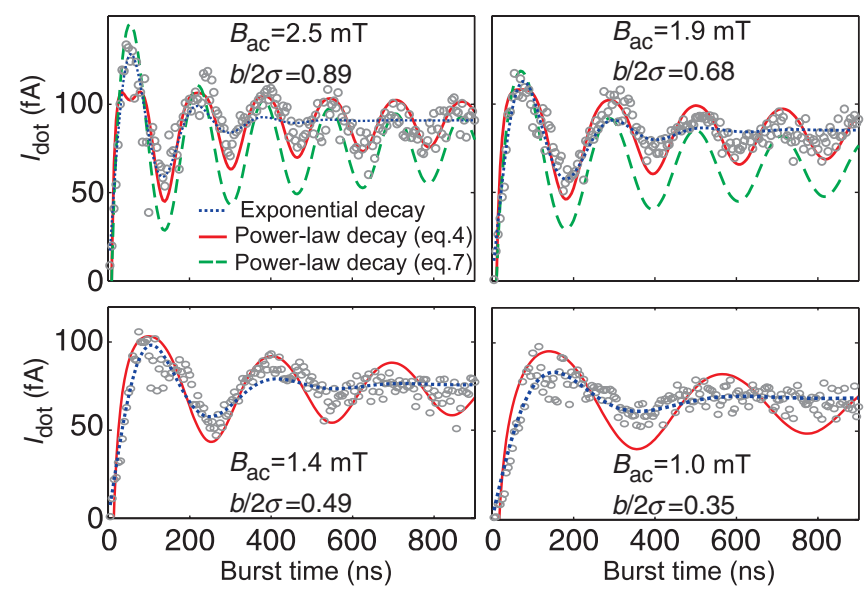

FIG. 1 (color online). Rabi oscillations for four different driving fields $B_{\mathrm{ac}}\left[B_{z}=55 \mathrm{mT}, g=0.355\right.$ and $\left.\sigma=g \mu_{B}(1.4 \mathrm{mT})\right]$. The gray circles represent the experimentally measured dot current (averaged over $15 \mathrm{~s}$ for each value of $t$ ), which reflects the probability to find an odd spin-parity state after the rf burst that generates $B_{\text {ac }}$. The dotted, solid, and dashed lines represent the best fit to the data of an exponentially decaying cosine function and the derived analytical expressions for $P_{\text {odd }}(t)$ and $P_{\text {odd }}^{(1)}(t)$ [Eqs. (4) and (7)], respectively. For clarity, the dashed line is shown only for the top two panels. The fit was carried out for the range 60 to $900 \mathrm{~ns}$ and the displayed values for $B_{\mathrm{ac}}$ were obtained from the fit with $P_{\text {odd }}(t)$ [Eq. (4)]. We fit the data with an exponentially decaying cosine with a tunable phase shift that is zero at $t=0: a_{1} e^{-t / a_{2}}\left[\cos (\phi)-\cos \left(2 \pi t / a_{3}+\phi\right)\right]+a_{4}(1-$ $\left.e^{-t / a_{2}}\right)$. The last term was added such that the saturation value is a fit parameter as well. We note that the fit is best for $\phi=\pi / 4$, as discussed in the text.
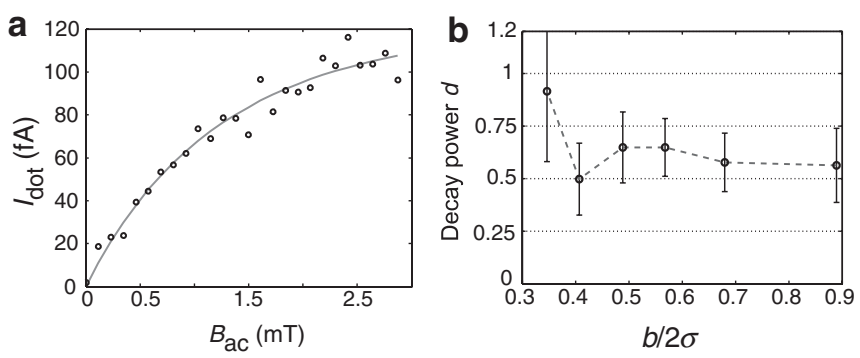

FIG. 2. (a) Dot current after an rf burst of $950 \mathrm{~ns}$ as a function of $B_{\text {ac }}$, approximately representing the steady-state value. The solid curve is the best fit with $a_{1}\left(\frac{1}{2}-2 C^{2}\right)$ : the steady-state expression of Eq. (4) with $a_{1}$ and $\sigma$ as fit parameters. We find, for the $95 \%$ confidence interval, $\sigma=g \mu_{B}(1.0-1.7 \mathrm{mT})$. (b) Decay power obtained from the best fit of the data (partially shown in Fig. 1) with the expression $a_{1}+a_{2} \cos \left(2 \pi t / a_{3}+\right.$ $\pi / 4) / t^{d}$, where $a_{1,2,3}$ and $d$ are fit parameters.

There might, however, still be a small difference in $h_{0}$ between the two dots, which we cannot determine quantitatively because the two models describe only two limiting cases. If present, such a difference in $h_{0}$ could help explain the small deviation between data and model at the first oscillation for $B_{\mathrm{ac}}=2.5 \mathrm{mT}$. It could originate from asymmetric feedback of the electron spins on the respective nuclear-spin baths, e.g., due to unequal dot sizes, leading to different hyperfine coupling constants.

Another observation is that for small driving fields, $B_{\mathrm{ac}}<1.9 \mathrm{mT}$, we see that the damping is faster than predicted. Possible explanations for this effect are corrections due to electron-nuclear flip-flops (transverse terms in the hyperfine Hamiltonian) or electric-field fluctuations. Electron-nuclear flip-flops may become relevant on a time scale $\sim \epsilon_{z} / \sigma^{2} \sim 1 \mu \mathrm{s}$ in this experiment. Electricfield fluctuations can couple to spin states via the spin-orbit interaction [27] or a finite electric-field dependent exchange coupling.

We continue the discussion with the experimental observation of the second theoretically predicted prominent feature of the Rabi oscillations, i.e., a phase shift of $\pi / 4$ in the oscillations, which is independent of all parameters. The value of $\phi$ can be extracted most accurately from the oscillations measured for a wide range and small steps of $B_{\mathrm{ac}}$, like the data shown in Fig. 3(a). That is because the Rabi period $T_{\text {Rabi }}=2 \pi / g \mu_{B}\left(\frac{1}{2} B_{\text {ac }}\right)=2 \pi / g \mu_{B}\left(\frac{1}{2} K I_{s}\right)$ contains only one unknown parameter $K$ (current to oscillating field amplitude $B_{\text {ac }}$ conversion factor, in units of $\mathrm{T} / \mathrm{A}$ ) which is independent of the current through the wire $I_{s}$ that generates $B_{\mathrm{ac}}$ [18]. The presence of a phase shift is visible in Fig. 3(a), where the green and blue lines correspond, respectively, to the maxima of a cosine with and without a phase shift of $\pi / 4$. The green lines match very well the yellow bands representing high data values. In contrast, the blue lines are located on the right side of the yellow bands for small burst times and more and more on the left side of the bands for increasing burst times. Thus, a 


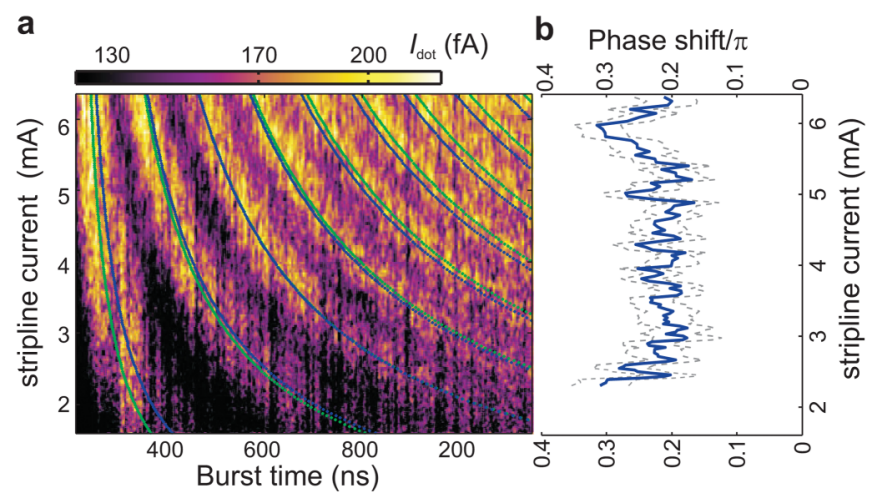

FIG. 3 (color). (a) The dot current (represented in color scale) is displayed over a wide range of $B_{\text {ac }}$ (the sweep axis) and burst durations. The green and blue lines correspond, respectively, to the maxima of a cosine with and without a phase shift of $\pi / 4$. The current-to-field conversion factor $K$ is fitted for both cases separately $(K=0.568 \mathrm{mT} / \mathrm{mA}$ and $K=0.60 \mathrm{mT} / \mathrm{mA}$ for, respectively, with and without phase shift; the fit range is $t=$ 60-500 ns and $I_{s}=3.6-6.3 \mathrm{~mA}$ ). (b) Phase shift for a wide range of $B_{\text {ac }}$, displayed as a function of stripline current $I_{s}$. Values obtained from a fit of each trace of the data in (a) (varying burst time, constant $\left.B_{\mathrm{ac}}\right)$ to a damped cosine $a_{1}-$ $a_{2} \cos \left(\frac{1}{2} K I_{s} g \mu_{B} t+a_{3} \pi\right) / \sqrt{t}$, where $a_{1,2,3}$ are fit parameters and $K=0.568 \mathrm{mT} / \mathrm{mA}$. $I_{s}$ is a known value in the experiment, extracted from the applied rf power. The gray dashed lines represent the $95 \%$ confidence interval.

cosine without a phase shift does not match with the observed Rabi oscillations.

In order to determine $\phi$ quantitatively, we perform a single two-dimensional fit of the complete data set in Fig. 3(a) with $P_{\text {odd }}(t)$ [Eq. (4)], excluding the $1 / t$ term (see [24]). The fit range is $t=100-900 \mathrm{~ns}$, such that the contribution from the $1 / t$ term of Eq. (4) can be neglected. For the $95 \%$ confidence interval we find $\phi=(0.23 \pm$ $0.01) \pi$, close to the theoretical value. The relation between $\phi$ and $B_{\text {ac }}$ is visible in Fig. 3(b), where we find no significant dependence of $\phi$ as a function of $B_{\mathrm{ac}}$, although the accuracy decreases for smaller $B_{\text {ac }}$ (values obtained from fits to single traces, see caption). We have not compensated for the effects of the finite rise time ( $<2 \mathrm{~ns}$ ) of the bursts, which leads to a small negative phase shift, on top of the expected positive $\pi / 4$ shift.

To conclude, we have experimentally observed a powerlaw decay and universal phase shift of driven single electron-spin oscillations. These features are theoretically understood by taking into account the coupling of the spin to the nuclear-spin bath, which is static on the time scale of the electron-spin evolution time. These reported results affect the prospect of making electron-spin based qubits in GaAs quantum dots. Namely, the slow power-law decay allows spin manipulation with smaller driving fields, and knowledge of the phase shift is relevant for determination of the correct pulse lengths. Furthermore, nonexponential coherence decay will affect error-correction schemes which usually account for exponential decays. For future investigation, it remains interesting to obtain more information about the nonstatic contributions of the nuclear bath or other possible decoherence mechanisms. This requires measuring the driven oscillations at larger external fields, with larger driving powers and longer evolution times than accessible in this work.

We thank T. Meunier, R. Hanson, Y. V. Nazarov and I. T. Vink for discussions; R. Schouten, A. van der Enden, R. Roeleveld, and W. den Braver for technical assistance. We acknowledge financial support from the Dutch Organization for Fundamental Research on Matter (FOM), the Netherlands Organization for Scientific Research (NWO), JST ICORP, NCCR Nanoscience, and the Swiss NSF.

[1] F. Bloch, Phys. Rev. 70, 460 (1946).

[2] D. P. DiVincenzo and D. Loss, Phys. Rev. B 71, 035318 (2005).

[3] J. Taylor and M. Lukin, Quant. Info. Proc. 5, 503 (2006).

[4] G. Ithier et al., Phys. Rev. B 72, 134519 (2005).

[5] A. V. Khaetskii and Y. V. Nazarov, Phys. Rev. B 61, 12639 (2000).

[6] V. N. Golovach, A. Khaetskii, and D. Loss, Phys. Rev. Lett. 93, 016601 (2004).

[7] J. M. Elzerman et al., Nature (London) 430, 431 (2004).

[8] M. Kroutvar et al., Nature (London) 432, 81 (2004).

[9] S. Amasha et al., arXiv:cond-mat/0607110.

[10] A. V. Khaetskii, D. Loss, and L. Glazman, Phys. Rev. Lett. 88, 186802 (2002).

[11] I. A. Merkulov, A. L. Efros, and M. Rosen, Phys. Rev. B 65, 205309 (2002)

[12] W. A. Coish and D. Loss, Phys. Rev. B 70, 195340 (2004).

[13] W. A. Coish and D. Loss, Phys. Rev. B 72, 125337 (2005).

[14] A. C. Johnson et al., Nature (London) 435, 925 (2005).

[15] F. H. L. Koppens et al., Science 309, 1346 (2005).

[16] J. R. Petta et al., Science 309, 2180 (2005).

[17] E. A. Laird et al., Phys. Rev. Lett. 97, 056801 (2006).

[18] F. H. L. Koppens et al., Nature (London) 442, 766 (2006).

[19] W. Yao, R.-B. Liu, and L.J. Sham, Phys. Rev. B 74, 195301 (2006).

[20] R. de Sousa and S. Das Sarma, Phys. Rev. B 67, 033301 (2003).

[21] D. Klauser, W. A. Coish, and D. Loss, Phys. Rev. B 73, 205302 (2006).

[22] C. Deng and X. Hu, Phys. Rev. B 73, 241303(R) (2006).

[23] C. Deng and X. Hu, Phys. Rev. B 74, 129902(E) (2006).

[24] See EPAPS Document No. E-PRLTAO-99-027736 for fit procedures and asymptotic expansion. For more information on EPAPS, see http://www.aip.org/pubservs/ epaps.html.

[25] V. V. Dobrovitski, H. A. De Raedt, M. I. Katsnelson, and B. N. Harmon, Phys. Rev. Lett. 90, 210401 (2003).

[26] R.-S. Huang, V. Dobrovitski, and B. Harmon, arXiv:condmat/0504449.

[27] M. Borhani, V. N. Golovach, and D. Loss, Phys. Rev. B 73, 155311 (2006). 